Article

\title{
Metal-Catalyzed Degradation of Cellulose in Ionic Liquid Media
}

\author{
Tiina Aid *(1), Mihkel Koel, Margus Lopp and Merike Vaher \\ Department of Chemistry and Biotechnology, Division of Chemistry, Tallinn University of Technology, \\ Akadeemia tee 15, 12618 Tallinn, Estonia; mihkel.koel@ttu.ee (M.K.); margus.lopp@ttu.ee (M.L.); \\ merike.vaher@ttu.ee (M.V.) \\ * Correspondence: tiina.aid@ttu.ee
}

Received: 14 June 2018; Accepted: 8 August 2018; Published: 10 August 2018

\begin{abstract}
Biomass conversion to 5-hydroxymethylfurfural (HMF) has been widely investigated as a sustainable alternative to petroleum-based feedstock, since it can be efficiently converted to fuel, plastic, polyester, and other industrial chemicals. In this report, the degradation of commercial cellulose, the isomerization of glucose to fructose, and the conversion of glucose to HMF in 1-butyl-3-methylimidazolium chloride ([BMIM]Cl]) using metal catalysts $\left(\mathrm{CrCl}_{3}, \mathrm{ZnCl}_{2}, \mathrm{MgCl}_{2}\right)$ as well as tungsten and molybdenum oxide-based polyoxometalates (POM) were investigated. Tungsten and molybdenum oxide-based POMs in ionic liquids (IL) were able to degrade cellulose to majority glucose and epimerize glucose to mannose (in the case of the molybdenum oxide-based POM). A certain amount of glucose was also converted to HMF. The tungsten oxide-based POM in IL showed good activity for cellulose degradation but the overall products yield remained $28.6 \%$ lower than those obtained using $\mathrm{CrCl}_{3}$ as a catalyst. Lowering the cellulose loading did not significantly influence the results and the addition of water to the reaction medium decreased the product yields remarkably.
\end{abstract}

Keywords: biorefinery; platform chemicals; cellulose degradation; catalysis

\section{Introduction}

An efficient utilization of renewable biomass resources, particularly lignocellulosic biomass, is important from the viewpoint of the production of industrial platform chemicals and fuels (Figure 1) [1]. An appropriate technology would efficiently deconstruct the biomass to release cellulose and hemicellulose and hydrolyze cellulosic components to generate oligosaccharides [2].

Of the fundamental building blocks from biorefineries, 5-hydroxymethylfurfural (HMF) is considered a key intermediate for the development of biomass-based products, because a series of compounds such as organic acids, polymer precursors, and biofuels derive from it [3].

Hence the selective catalytic conversion of cellulose to a platform chemical such as glucose, HMF, sorbitol or gluconic acid under mild conditions is the most desirable route in industry $[4,5]$. However, a high-yield, low-cost, energy-efficient, and direct conversion method for cellulose to HMF is still a challenge to researchers in the field.

Ionic liquids (ILs) are being investigated as effective low-impact solvents for the conversion of cellulose due to being relatively easy to handle, recyclable, and in possession of a negligible vapor pressure. Moreover, ILs have an ability to solubilize low-toxicity metal catalysts for the direct conversion of cellulose to value-added chemicals. However, most published papers focus mainly on the advantages of ionic liquids in the separation of cellulose from lignocellulosic biomass over traditional methods [6-9]. 


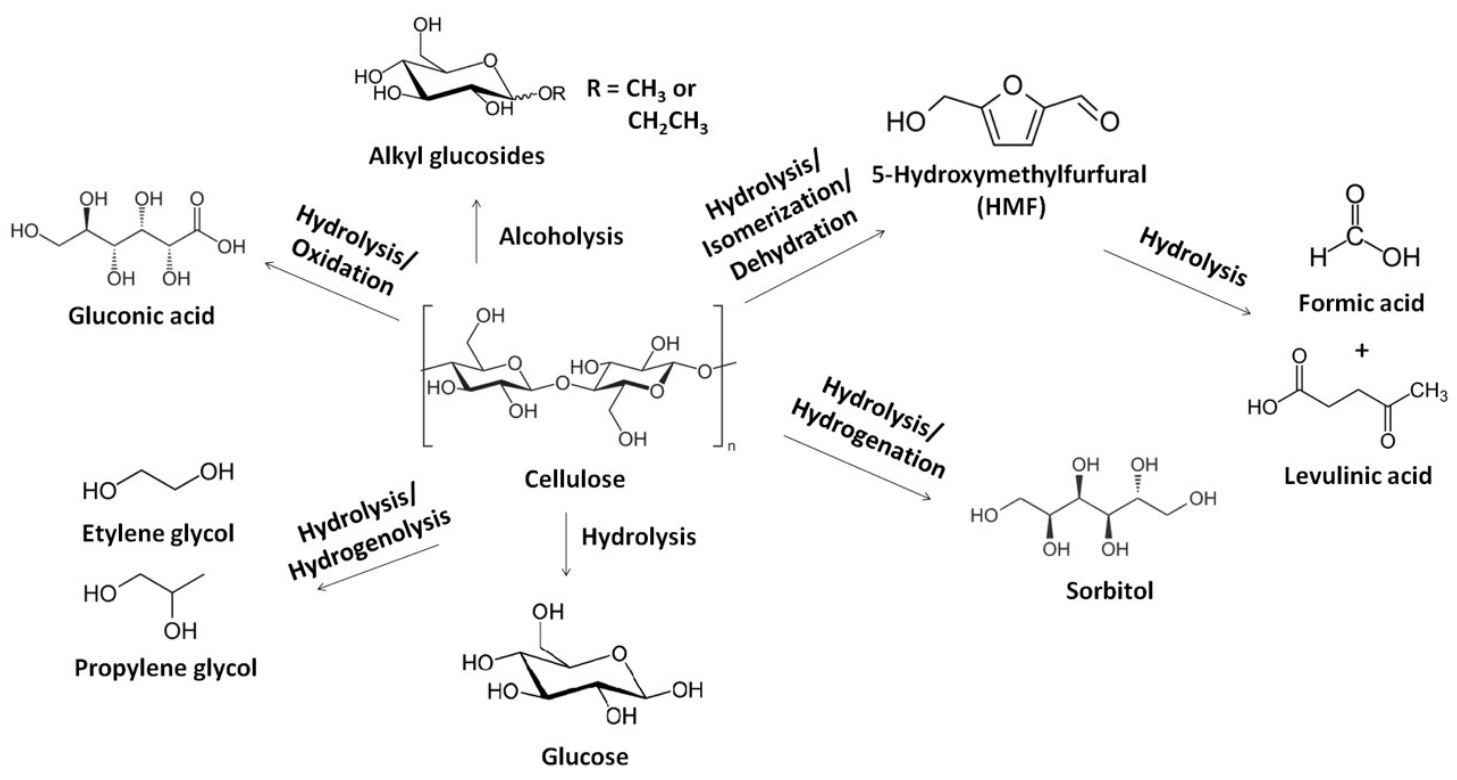

Figure 1. Typical platform chemicals produced from the catalytic transformations of cellulose.

From previous studies it appears that when using cellulose as a feedstock for HMF formation, the key steps are the dissolution and depolymerisation of cellulose to glucose monomers and not the isomerization to fructose and subsequent dehydration of fructose to HMF. Cellulose has an abundant number of intra- and intermolecular hydrogen bonds that make it intrinsically recalcitrant to depolymerize which is why ionic liquids as solvents can provide a solution due to their specific ability to dissolve the compound [10]. ILs appear to be even more advantageous in overcoming these problems as they can act as both solvents and catalysts [7]. The catalytic conversion of biomass-derived carbohydrates to value-added chemicals is a commercially important reaction and requires the use of both Lewis and Brønsted acids. Multifunctional ILs with both types of acidity are promising catalysts as well as solvents for the one-pot conversion of glucose to value-added chemicals.

Until now, the production of HMF with glucose as a feedstock has efficiently used chromium chloride catalyst together with ionic liquids. Zhao et al. [11] were the first to report an unparalleled yield of $69 \% \mathrm{HMF}$ catalyzed by chromium (II) chloride $\left(\mathrm{CrCl}_{2}\right)$ in a medium of 1-ethyl-3-methylimidazolium chloride ([EMIM]Cl) at $100{ }^{\circ} \mathrm{C}$ for $3 \mathrm{~h}$. Recently, the dehydration of glucose to $\mathrm{HMF}$ with a yield of about $67 \%$ was achieved in [EMIM]Cl and $N, N$-dimethylacetamide (DMA) by using $\mathrm{CrCl}_{2}$ or $\mathrm{CrCl}_{3}$ at $100{ }^{\circ} \mathrm{C}$ for $6 \mathrm{~h}$ [12]. Dehydration of cellulose to HMF with a yield of about $89 \%$ was achieved in [EMIM]Cl by using $\mathrm{CrCl}_{2}$ at $120^{\circ} \mathrm{C}$ for $6 \mathrm{~h}$ [13]).

The catalytic activity of a $\mathrm{ZnCl}_{3}$-containing IL was found to be highly Lewis acidic, and was the controlling parameter for glucose conversion, resulting in yields of $13.4 \%, 23.8 \%$, and $67.1 \%$ of HMF, levulinic acid (LA) and formic acid (FA), respectively. These multifunctional chlorometallate ILs were found to be recyclable with no loss of metal chloride from their anion [14].

Brønsted acidic ionic liquids (BAILs) showed a very good catalytic effect on the conversion of raw biomass to $C 5$ sugars and furfural. In water medium the remarkably high yield of $C 5$ sugars from bagasse, $88 \%$, was obtained using 1-methyl-3(3-sulfopropyl)-imidazolium hydrogen sulfate $\left(\left[\mathrm{C}_{3} \mathrm{SO}_{3} \mathrm{HMIM}\right]\left[\mathrm{HSO}_{4}\right]\right)$. Similarly, with this BAIL, a high yield (73\%) of furfural was obtained in the one-pot method using a water/toluene biphasic solvent system [15].

Calculations revealed the dependence of the catalytic performance of $\left[\mathrm{C}_{3} \mathrm{SO}_{3} \mathrm{HMIM}\right]\left[\mathrm{HSO}{ }_{4}\right]$ BAIL on the acidity and nucleophilicity of its constituent ions [16].

1,1,3,3-tetramethylguanidine tetrafluoroborate $\left([\mathrm{TMG}] \mathrm{BF}_{4}\right)$ as a solvent was confirmed to exhibit excellent catalytic activity in the conversion of $\mathrm{C} 6$ carbohydrates to HMF. The HMF yields from the 
conversion of fructose, glucose, cellobiose, and microcrystalline cellulose (MCC) were $74 \%, 27 \%, 20 \%$, and $18 \%$, respectively [17].

The search for effective catalysts has brought polyoxometalates-anionic metal oxides under consideration because of their unique properties, such as strong Brønsted acidity, good oxidizing ability, high water tolerance, low corrosiveness, and recoverability. In addition, POMs have exhibited a promising performance in the transformation of cellulose into platform chemicals in both homogeneous and heterogeneous systems [18].

A review by Deng et al. [19] highlights the following good catalytic properties of POMs in the conversion of cellulose to platform chemicals:

1. $\quad$ strong Brønsted acidity;

2. the capability to activate oxidants such as $\mathrm{O}_{2}$ and $\mathrm{H}_{2} \mathrm{O}_{2}$ for selective oxidation;

3. high water tolerance;

4. tunable acidity, redox potential, and solubility in various media, which allow the rational design of active sites on molecular and atomic scales;

5. high thermal and oxidative stability as compared with common molecular catalysts such as organometallic complexes and enzymes;

6. ease of handling and separation, and the relatively low corrosiveness, possibly owing to the generated corrosion-inhibiting films, which allow them to act as environmentally friendly liquid-phase catalysts, unlike mineral acids.

Another approach is to use task-specific ionic liquids with polyoxometalates (POMs) contained in them. Due to their high negative charge and large metal-oxide framework, POMs can react with a variety of cationic organic groups to form novel functional ionic liquids, POMs-ILs. IL cations with various structures and properties can provide organic blocks to modify POM catalysts, thanks to their acidity, polarity, solubility, redox properties, and surface structures.

Polyoxomethalates-based ionic liquids (POM-IL) as solid acid catalysts have been used for the direct conversion of fructose to HMF. The phosphotungstic acid (HPW)-derived ionic liquid shows the highest catalytic performance (up to $99 \%$ of the yield) in the formation of HMF. In this study, the catalyst afforded a good yield of HMF from inulin (76\%) and sucrose (45\%) as well [20].

In another work [21], POM-IL were synthesized and employed for the one-pot dissolution and conversion of powdered switchgrass biomass. For comparison purposes, Avicel Cellulose was also treated under identical conditions. The most promising for biomass conversion was found to be the combination of phosphotungstic acid hydrate and 1-butyl-3-methylimidazolium bromide. Avicel Cellulose was then utilized for the hydrolysis at $200{ }^{\circ} \mathrm{C}$ for $120 \mathrm{~min}$, and as a result, approximately $31 \mathrm{wt} \%$ of the biomass and $13 \mathrm{wt} \%$ of Avicel Cellulose were converted to water-soluble products; i.e., sugars obtained from the deconstructed cellulose [21].

There are some other examples where heteropolyacids act as efficient catalysts for the conversion of glucose. For example, a 98\% conversion of glucose to HMF in ionic liquids and a $99 \%$ selectivity of HMF were attained after a $3 \mathrm{~h}$ reaction time at $393 \mathrm{~K}$ by using 12-molybdophosphoric acid in a mixture of either 1-ethyl-3-methylimidazolium or 1-butyl-3-methylimidazolium chloride with acetonitrile as a co-solvent [22].

Analysis of ILs-containing samples where capillary electrophoresis (CE) is proposed as an effective method for the simultaneous determination of mono-, di- and oligosaccharides, as well as HMF and organic acids (acetic and levulinic acids can be formed during degradation of HMF), is no easy task $[23,24]$. The objective of this case study was to compare molybdenum and tungsten oxide-based POM-ILs catalytic performance with metal catalysts, such as $\mathrm{CrCl}_{3}, \mathrm{ZnCl}_{2}$ and $\mathrm{MgCl}_{2}$ using ILs as solvents. 


\section{Results and Discussion}

The main goal was to monitor the transformation of microcrystalline cellulose to HMF via glucose and fructose by different widely-studied catalysts and compare the results with molybdenum and tungsten oxide-based POMs-ILs. Although the isomerization and epimerization of glucose might occur, the formation of fructose predominated. The formation of fructose via isomerization required some reorganization of the intermediate, whereas the formation of mannose is reported through rotation around the $\mathrm{C} 2-\mathrm{C} 3$ bond [25]. One of the glucose isomerization reaction pathways is analogous to that observed in metalloenzymes, such as D-xylose isomerase (XI), which contain two divalent Lewis acid metal centers $\left(\mathrm{Mg}^{2+}\right.$ or $\left.\mathrm{Mn}^{2+}\right)$ confined within a hydrophobic pocket [26,27]. The reaction pathway includes the ring-opening of glucose and coordination with glucose $\mathrm{O} 1$ and $\mathrm{O} 2$ atoms prior to the isomerization of the ring-opened glucose chain from position $\mathrm{C} 2$ to $\mathrm{C} 1$ (via 1,2 intramolecular hydride shift) [28].

Table 1 (Table S1) shows, the conversion of cellulose to HMF in [BMIM] Cl at $100{ }^{\circ} \mathrm{C}$ for $24 \mathrm{~h}$ with $\mathrm{ZnCl}_{2}, \mathrm{MgCl}_{2}, \mathrm{CrCl}_{3}$, molybdenum and tungsten oxide-based POM-ILs as catalysts. In the case of molybdenum and tungsten oxides-based POM-ILs the main products were monosaccharides. Table 1 also contains the total product yields where all of the obtained products are summarized.

Table 1. Glucose, mannose and 5-hydroxymethylfurfural (HMF) contents in cellulose samples degraded in [BMIM]Cl using $\mathrm{ZnCl}_{2}, \mathrm{MgCl}_{2}, \mathrm{CrCl}_{3}$, tungsten (W-POM) and molybdenum (Mo-POM) oxide-based polyoxometalates (POMs) as catalysts. Catalyst loading was $10 \mathrm{wt} \%$ from cellulose and cellulose loading was $10 \mathrm{wt} \%$.

\begin{tabular}{ccccc}
\hline Catalyst & Glucose, \% & Mannose, \% & HMF, \% & Total Products Yield, \% \\
\hline No catalyst & $>0.04$ & $>0.04$ & $>0.04$ & $>0.04$ \\
$\mathrm{ZnCl}_{2}$ & $>0.04$ & $>0.04$ & 4.64 & 4.64 \\
$\mathrm{MgCl}_{2}$ & $>0.04$ & $>0.04$ & 3.78 & 3.78 \\
$\mathrm{CrCl}_{3}$ & $>0.04$ & $>0.04$ & 55.3 & 55.3 \\
$\mathrm{~W}-\mathrm{POM}$ & 33.7 & $>0.04$ & 5.82 & 39.5 \\
Mo-POM & 4.96 & 2.26 & 2.95 & 10.2 \\
\hline
\end{tabular}

As expected, the sample with no catalyst did not contain any of the investigated products. The tungsten oxide-based POM showed good activity for cellulose degradation, however, the overall yield of products (glucose and HMF) remained $29 \%$ lower than that obtained with $\mathrm{CrCl}_{3}$ as a catalyst. The main product formed was glucose instead of HMF. The molybdenum oxide-based POM was the only catalyst that was able to epimerize glucose to mannose, while the latter accounted for $22 \%$ of the products obtained. The catalytic activity of the molybdenum oxide-based POM remained remarkably lower and the overall product yields (glucose, mannose and $\mathrm{HMF}$ ) were $82 \%$ less compared to $\mathrm{CrCl}_{3}$. The catalytic ability of $\mathrm{MgCl}_{2}$ and $\mathrm{ZnCl}_{2}$ were the lowest and $\mathrm{HMF}$ yields were $6.8 \%$ and $8.4 \%$ of that obtained with $\mathrm{CrCl}_{3}$, respectively.

Some metal chlorides are very effective as catalysts for the synthesis of HMF in ILs [29,30]. The isomerization of glucose into fructose is favored by the presence of Lewis acid sites [25]. Figure 2 shows the mechanism proposed by Zhou et al. for the interaction of metal chlorides with glucose in ILs such as [BMIM]Cl. According to this mechanism, good catalytic performance of $\mathrm{CrCl}_{3} \cdot 6 \mathrm{H}_{2} \mathrm{O}$ may be explained by the formation of a more stable metal chloride-glucose complex due to the stronger coordination ability of $\mathrm{Cl}^{-}$with a chromium center [29-31].

$\mathrm{Li}$ et al. [32] demonstrated in their study that according to density functional theory (DFT) calculations, isomerization of glucose to fructose over tungsten oxide-based catalysts is possible because of Lewis acid sites (W6+), terminal W-oxo groups that are Lewis basic sites, and proton mediators, such as "structural" and physisorbed water on the oxide surface. According to their study the key aspect of the catalytic mechanism is the proton shift from $\mathrm{C} 2 \rightarrow \mathrm{C} 1$ that is promoted by a synergistic action of the Lewis acid sites, which is followed by a proton-transfer [32]. 
Ju et al. [33] demonstrated in their ${ }^{13}$ C NMR study that molybdenum-based POMs are active and selective catalysts for the epimerization of aldoses. The epimerization mechanism involves electron transfer from the aldose to the molybdenum oxide octahedra surface units of the POM followed by an intra molecular $\mathrm{C} 1 \rightarrow \mathrm{C} 2$ carbon shift. They also report that replacing Mo with $\mathrm{W}$ in the Kegging structure POM resulted in loss of the epimerization activity, indicating that the molybdenum octahedral located in the cagelike structure of the POM play an important role in activating the epimerization of glucose [33].

Nguyen et al. [34] demonstrated in their ${ }^{13} \mathrm{C}$ NMR and ${ }^{1} \mathrm{H}$ NMR study that glucose epimerization to mannose using Lewis acids, such as $\mathrm{MCl}_{3}$ in aqueous phase $\left(\mathrm{CrCl}_{3}\right.$ was also tested), proceeds via two parallel mechanisms: first a reverse $\mathrm{C} 2 \rightarrow \mathrm{C} 1$ hydride transfer followed by a $\mathrm{C} 1 \rightarrow \mathrm{C} 2$ intramolecular carbon shift. They also report that $\mathrm{MCl}_{3}$ are also able to epimerize glucose to mannose in low yields and since fructose formation is predominant, the hydride transfer is the more dominant pathway of glucose conversion [34].

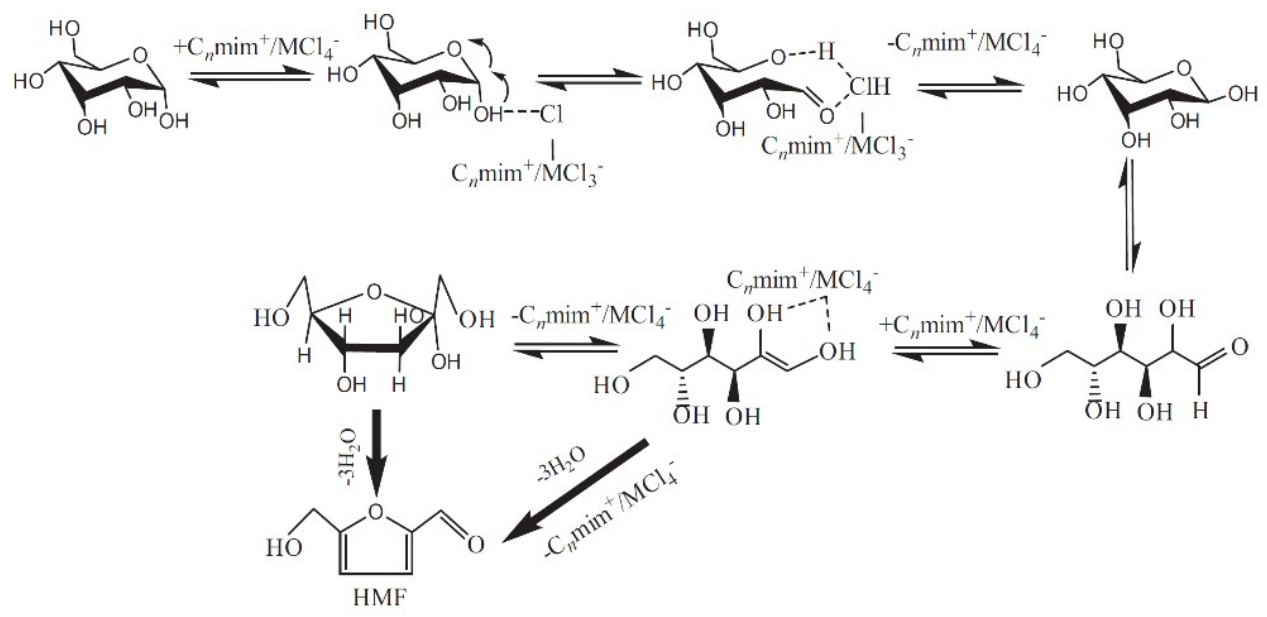

Figure 2. Interaction of metal chlorides with glucose to produce $\mathrm{HMF}$ in $\mathrm{C}_{n} \mathrm{MIM} / \mathrm{MCl}_{4}(n=4$, 1-n-butyl-3-methylimidazolium, [BMIM]Cl; $\mathrm{M}=\mathrm{Cr}, \mathrm{Al}$ and $\mathrm{Fe}$ ) [35].

\subsection{Reaction Time for POM-IL Catalysts}

Figure 3 (Tables S2 and S3) shows the effect of time on the yield of glucose, mannose and HMF from cellulose at 100 and $120{ }^{\circ} \mathrm{C}$ for $72 \mathrm{~h}$ using tungsten and molybdenum oxide-based POMs as catalysts.

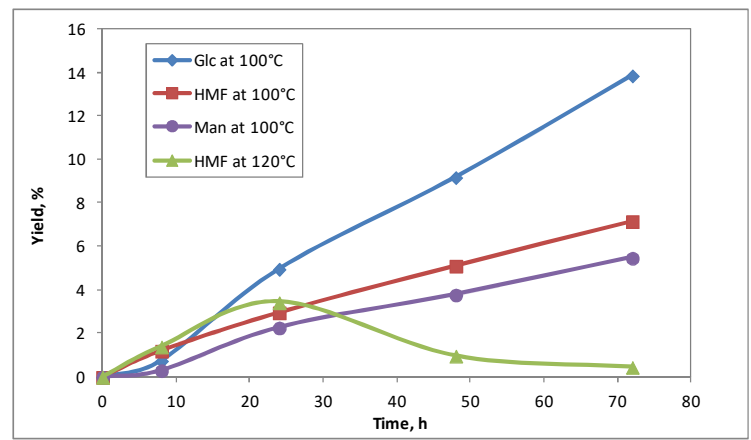

(a)

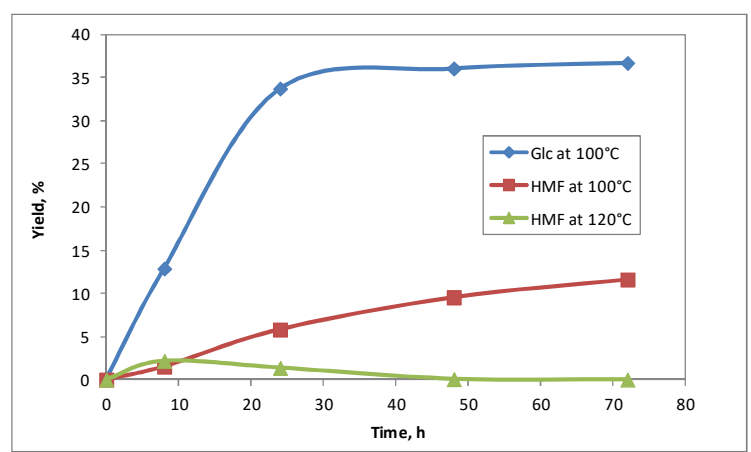

(b)

Figure 3. Conversion of cellulose to glucose, mannose and $\mathrm{HMF}$ in [BMIM]Cl at 100 and $120{ }^{\circ} \mathrm{C}$ by using POMs based on (a) molybdenum oxide and (b) tungsten oxide. Catalyst loading was $10 \mathrm{wt} \%$ from cellulose and cellulose loading was $10 \mathrm{wt} \%$ from solvent. 
In the case of the molybdenum oxide-based POM, prolonging the reaction time improved the $\mathrm{HMF}$ yield at $100^{\circ} \mathrm{C}$ and the plateau in its concentration was not achieved by $72 \mathrm{~h}$. The same trend held true for the yields of glucose and mannose. At $120^{\circ} \mathrm{C}$, glucose and mannose were not detected and the maximum HMF yield remained lower than the yield obtained at $100{ }^{\circ} \mathrm{C}$. The maximum $\mathrm{HMF}$ yield, which was achieved at $24 \mathrm{~h}$, was 52\% lower, and furthermore, it started to decrease with a longer reaction time. In the case of the tungsten oxide-based POM, prolonging the reaction time at $100{ }^{\circ} \mathrm{C}$ improved the glucose yield up to $24 \mathrm{~h}$ and then a plateau was achieved. The HMF yield increased slowly for $72 \mathrm{~h}$. Eminov et al. [7] reported that the highest $\mathrm{HMF}$ yield with $\mathrm{CrCl}_{3} \cdot 6 \mathrm{H}_{2} \mathrm{O}$ as a catalyst in [BMIM]Cl was obtained at $120^{\circ} \mathrm{C}$ and it was 5 times higher than the yield obtained at $100{ }^{\circ} \mathrm{C}$. In this work, the temperature was also increased by $20^{\circ} \mathrm{C}$, yet the ability to convert glucose to HMF was not improved. Instead, the opposite effect was observed: glucose was not present in any sample and the maximum HMF yield was achieved at $8 \mathrm{~h}$, remaining $81.4 \%$ lower than the result obtained at $100{ }^{\circ} \mathrm{C}$. According to the literature the lower yield could be caused by humin formation at higher temperatures [36]. Possible humin formation is also supported by the fact that a dark precipitate was formed when the higher temperature was used.

\subsection{Cellulose Loading and the Efficiency of POM Formation}

Chidambaram and Bell [22] reported that in [BMIM]Cl at $120{ }^{\circ} \mathrm{C} 3 \%$ of glucose is converted to humin even without using a catalyst. During the investigated process humins can be also formed due to the oligomerization of glucose or fructose with itself as well as with HMF. However, this is inhibiting the conversion of cellulose to shorter oligomers and glucose [36,37]. The formation of glucose and conversion to the other products must be balanced well to avoid the formation of humins. Thereby, cellulose loadings of 5 and $10 \mathrm{wt} \%$ were selected to investigate the effectivity of HMF formation at $120^{\circ} \mathrm{C}$.

Figure 4 (Table S4) shows the results for conversion of cellulose to $\mathrm{HMF}$ in [BMIM]Cl at $120{ }^{\circ} \mathrm{C}$ by using tungsten and molybdenum oxide-based POMs at $5 \mathrm{wt} \%$ and $10 \mathrm{wt} \%$ substrate loading. The HMF yield followed the same trend in spite of substrate loading for both the catalysts used. In the case of the molybdenum oxide-based POM-IL the maximum HMF yield was achieved at $24 \mathrm{~h}$ and was $52.2 \%$ of the maximum yield obtained with a $10 \mathrm{wt} \%$ catalyst loading. The maximum HMF yield for the tungsten oxide-based catalyst was achieved during $8 \mathrm{~h}$ and made $46.8 \%$ of the yield obtained with a $10 \%$ substrate loading. In addition, all samples obtained from 48 and $72 \mathrm{~h}$ experiments contained dark colored precipitate that was most probably humin. Lowering the substrate loading did not improve the conversion to HMF.

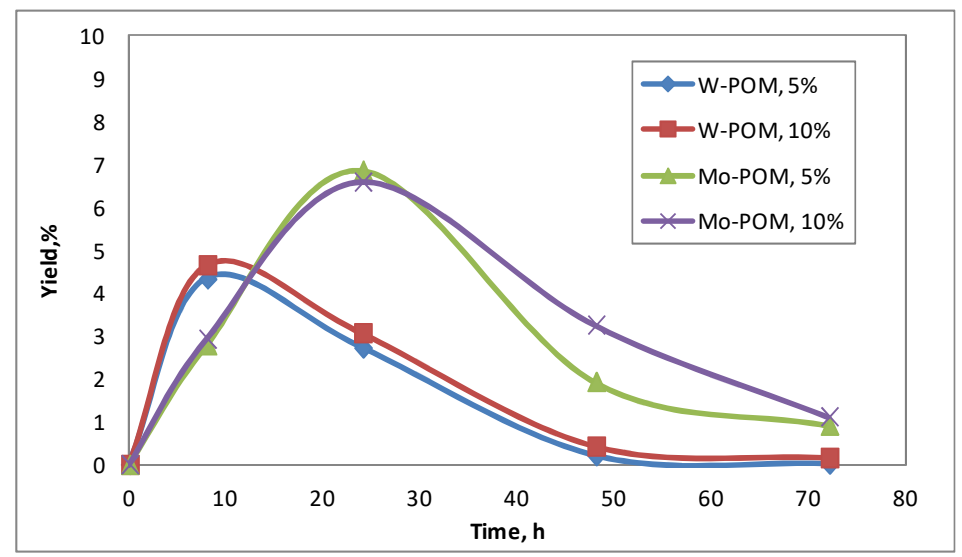

Figure 4. Conversion of cellulose to $\mathrm{HMF}$ in [BMIM]Cl at $120^{\circ} \mathrm{C}$ by using tungsten and molybdenum oxide-based POMs at 5 and $10 \%$ substrate loading. Catalyst loading was $10 \mathrm{wt} \%$ from cellulose and cellulose loading was 5 or $10 \mathrm{wt} \%$ from solvent. 
Figure 5 (Tables S5 and S6) shows the cellulose degradation at $100{ }^{\circ} \mathrm{C}$ for $72 \mathrm{~h}$ to evaluate the efficiency of POM formation in the IL medium. The catalyst (phosphotungstic acid hydrate) was added either $20 \mathrm{~min}$ before or $3 \mathrm{~h}$ after the substrate.

The highest glucose and HMF yields were when the catalyst was added to the sample before the substrate, with respective yields at $73.7 \%$ and $68.4 \%$ higher. These results show that the formation of POM is more efficient when the catalyst was added before the substrate.

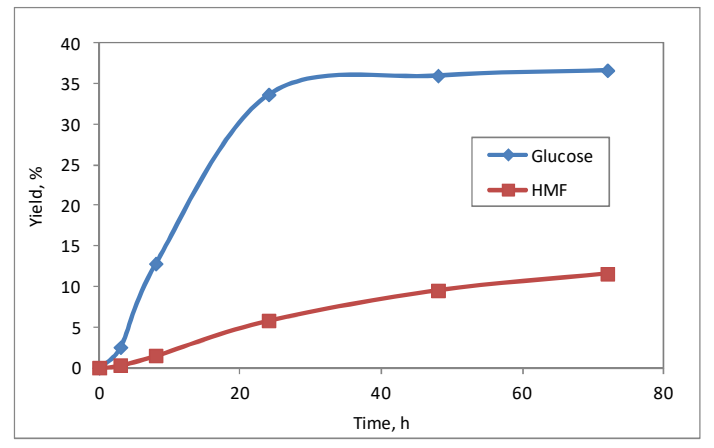

(a)

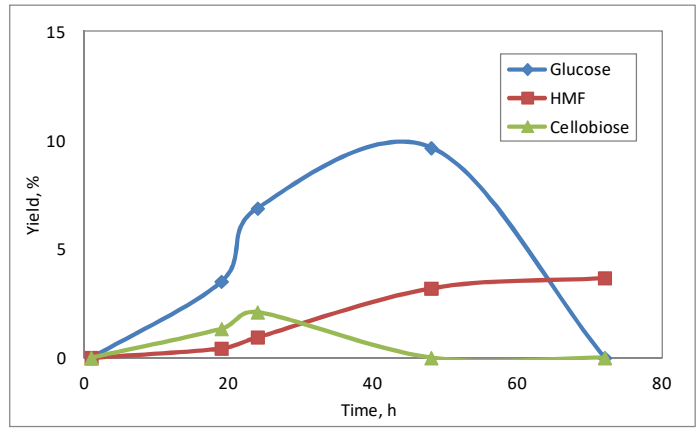

(b)

Figure 5. Efficiency of cellulose degradation in [BMIM]Cl at $100{ }^{\circ} \mathrm{C}$ by using phosphotungstic acid hydrate added (a) 20 min before the substrate, (b) $3 \mathrm{~h}$ after the substrate. Catalyst loading was $10 \mathrm{wt} \%$ from cellulose and cellulose loading was $10 \mathrm{wt} \%$ from solvent.

\subsection{Effect of the Water Content of the Reaction Medium on the Conversion of Cellulose to HMF}

Some data is available from the literature on metal catalysts. Zhang et al. [10] reported an increase of HMF yield from glucose in the presence of a higher amount of water in [EMIM]Cl when $\mathrm{CrCl}_{2} \mathrm{was}$ used as a catalyst. From the other side, Zhao et al. [12] reported that water has no influence on reaction yields. No data was available for POM-s. Since [BMIM]Cl is a hydroscopic ionic liquid, the influence of water content on the reaction dynamics by using tungsten oxide-based POM as catalysts was studied (Figure 6, Tables S7 and S8). The efficiency of HMF conversion and the influence of the reaction medium water content on the process were evaluated at $100{ }^{\circ} \mathrm{C}$ for $72 \mathrm{~h}$.

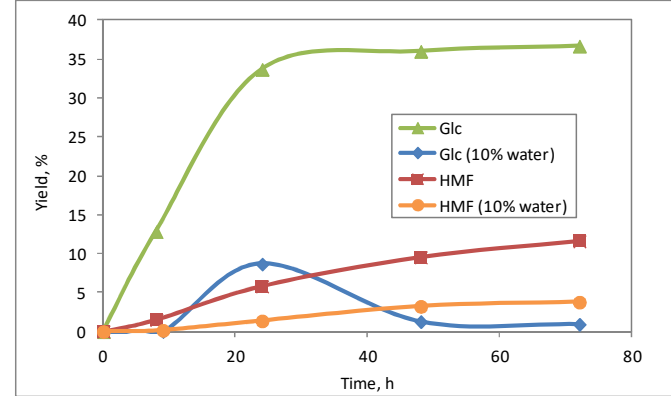

(a)

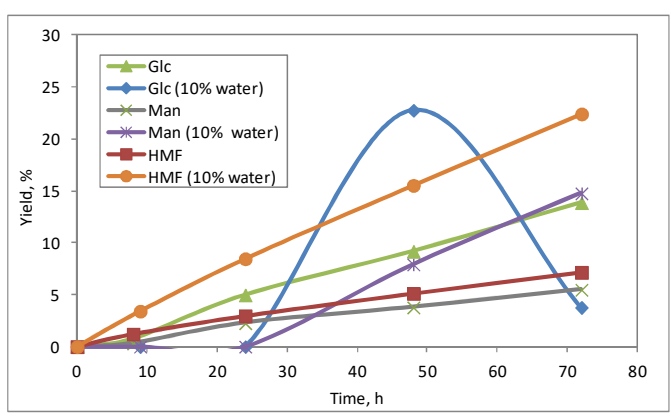

(b)

Figure 6. Conversion of cellulose to glucose, mannose and $\mathrm{HMF}$ in $[\mathrm{BMIM}] \mathrm{Cl}$ at $100{ }^{\circ} \mathrm{C}$ in the presence of water (10 wt \% of solvent) by using POMs based on (a) tungsten oxide, (b) molybdenum oxide. Catalyst loading was $10 \mathrm{wt} \%$ from cellulose and cellulose loading was $10 \mathrm{wt} \%$ from solvent.

Tungsten (VI) and molybdenum (VI) are expected to form POMs with imidazolium-based ILs. POMs are a class of anionic metal-oxygen clusters built by the connection of [MO]x polyhedral of the early transition metals in their highest oxidation states [38].

In the case of the tungsten oxide-based POM, the highest glucose and HMF yields in the presence of water were respectively $76.3 \%$ and $67.4 \%$ lower. The same tendency was observed with the 
molybdenum oxide-based POM-IL: the yields of glucose, mannose and HMF were correspondingly $72.7 \%, 59.2 \%$ and $52.8 \%$ lower in the presence of water. The experiment showed that increasing the water content resulted in the loss of catalytic activity of both the catalysts used.

\section{Materials and Methods}

\subsection{Catalytic Formation of HMF from Cellulose}

All experiments were carried out under aerobic conditions using dried ionic liquids; however, IL was handled and weighed as quickly as possible to prevent further absorption of water. All the catalysts used were in their hydrated form. Catalysts $\mathrm{CrCl}_{3}, \mathrm{ZnCl}_{2}, \mathrm{MgCl}_{2}$, phosphotungstic acid hydrate, phosphomolybdic acid hydrate and microcrystalline cellulose were obtained from Sigma-Aldrich (Sigma-Aldrich, Steinheim, Germany) and were used as received. [BMIM]Cl was obtained from IoLiTec (Ionic Liquids Technologies $\mathrm{GmbH}$, Heilbronn, Germany) and was vacuum-dried before use. The IL water content, which was checked by Karl-Fischer-Titration, was $0.6-0.7 \%$. Catalyst loading was $10 \mathrm{wt} \%$ of the substrate in all cases, the substrate loading was $10 \mathrm{wt} \%$ from the solvent in most cases, if not otherwise stated, and the sample typically contained $1 \mathrm{~g}$ of IL. The experiments were carried out on a magnetic stirrer plate at 100 or $120^{\circ} \mathrm{C}$ in open flasks with constant stirring. All the concentrations of oligo- and monosaccharides and HMF in reaction media were determined by CE and were analyzed at least in triplicate [23]. At first, the catalyst was dissolved in IL and after 20 min the cellulose (substrate) was added to the reaction mixture. The samples to compare different catalysts (Table 1) were collected at $24 \mathrm{~h}$ after the addition of cellulose. Unusually, a long reaction time (considering the speed of $\mathrm{CrCl}_{3}$ catalyst) was chosen because preliminary testing showed a slow conversion for molybdenum and tungsten oxide-based POMs.

\subsection{Analysis}

All the samples were analyzed by capillary electrophoresis and the yields were calculated based on calibration curves constructed using authentic standards. CE separations were performed using an Agilent 3D instrument (Agilent Technologies, Waldbronn, Germany) equipped with a diode array UV/Vis detector. Data acquisition and instrument control were carried out using Agilent Technologies HP 3D Chemstation software. The separation was performed using a fused silica capillary with an effective length of $61.5 \mathrm{~cm}$ (total length $70 \mathrm{~cm}$ ) and ID of $22.5 \mu \mathrm{m}$ (Polymicro Technologies Inc., Wilmington, DE, USA). Capillary walls contained a semi-permanent coating with $\left[\mathrm{C}_{14} \mathrm{MIM}\right] \mathrm{Cl}$ that was added to the background electrolyte (BGE). The BGE was composed of $138 \mathrm{mM} \mathrm{NaOH}, 40 \mathrm{mM}$ maleic acid and $5 \mathrm{mM}\left[\mathrm{C}_{14} \mathrm{MIM}\right] \mathrm{Cl}$. The samples were injected hydrodynamically under a pressure of $50 \mathrm{mbar}$ for $20 \mathrm{~s}$ and the separations were performed at $25^{\circ} \mathrm{C}$ by using a voltage of $-21 \mathrm{kV}$. The detection wavelength was $210 \mathrm{~nm}$ in the case of carbohydrates and $270 \mathrm{~nm}$ for HMF. Before each run the capillary was filled with BGE for $7 \mathrm{~min}$ and between the runs it was flushed with $1 \mathrm{M} \mathrm{NaOH}$ for 2 min and ultrapure water for $3 \mathrm{~min}$. BGE was prepared on the first day and stored at room temperature for one month. All these conditions were developed in our previous study [23]. Standards used to construct calibration curves and BGE substances, namely D-(+)-glucose, D-(-)-fructose, D-(+)-mannose, $\mathrm{D}-(+)$-cellobiose, sucrose, $\mathrm{HMF}$, maleic acid and $\mathrm{NaOH}$, were obtained from Sigma-Aldrich and were used as received. $\left[\mathrm{C}_{14} \mathrm{MIM}\right] \mathrm{Cl}$ was obtained from IoLiTec and was vacuum dried before use.

\section{Conclusions}

It has been shown that tungsten and molybdenum oxide-based polyoxometalates were able to decompose cellulose. The main decomposition products were carbohydrates such as glucose and mannose. The ability of the polyoxometalates to convert glucose to 5-hydroxymethylfurfural remained low in the mild conditions used and the overall product yields with the use of the tungsten oxide-based polyoxometalates remained $28.6 \%$ lower compared to the yield obtained with $\mathrm{CrCl}_{3}$. It was expected that increasing the temperature would improve the conversion of HMF but, surprisingly, increasing 
the temperature by $20^{\circ} \mathrm{C}$ did not increase the HMF outcome. The increase of the water content of the reaction medium, an influencing factor in fructose dehydration, resulted in the loss of catalytic activity of tungsten and molybdenum oxide-based polyoxometalates.

Supplementary Materials: The following are available online at http:/ / www.mdpi.com/2304-6740/6/3/78/s1, Table S1: Glucose, mannose and HMF contents in cellulose samples degraded in [BMIM]Cl using $\mathrm{ZnCl}_{2}, \mathrm{MgCl}_{2}$, $\mathrm{CrCl}_{3}$, tungsten (W-POM) and molybdenum (Mo-POM) oxide-based POMs as catalysts, Table S2: Conversion of cellulose to glucose, mannose and $\mathrm{HMF}$ in $[\mathrm{BMIM}] \mathrm{Cl}$ at 100 and $120^{\circ} \mathrm{C}$ by using POMs based on molybdenum oxide, Table S3: Conversion of cellulose to glucose, mannose and HMF in [BMIM]Cl at 100 and $120^{\circ} \mathrm{C}$ by using POMs based on tungsten oxide, Table S4: Efficiency of cellulose degradation in [BMIM]Cl at $100^{\circ} \mathrm{C}$ by using phosphotungstic acid hydrate added 20 min before the substrate, Table S5: Conversion of cellulose to HMF in [BMIM]Cl at $120^{\circ} \mathrm{C}$ by using tungsten and molybdenum oxides-based POMs at 5 and $10 \%$ substrate loading, Table S6: Efficiency of cellulose degradation in [BMIM]Cl at $100^{\circ} \mathrm{C}$ by using phosphotungstic acid hydrate added $3 \mathrm{~h}$ after the substrate, Table S7: Conversion of cellulose to glucose, mannose and $\mathrm{HMF}$ in [BMIM]Cl at $100{ }^{\circ} \mathrm{C}$ in the presence of water (10 wt \% of solvent) by using POMs based on tungsten oxide, Table S8: Conversion of cellulose to glucose, mannose and HMF in [BMIM]Cl at $100{ }^{\circ} \mathrm{C}$ in the presence of water (10 wt \% of solvent) by using POMs based on (a) tungsten oxide, (b) molybdenum oxide.

Author Contributions: T.A. and M.V. conceived and designed the experiments; T.A. performed the experiments; T.A. and M.V. analyzed the data; M.V. and M.L. contributed reagents/materials/analysis tools. The manuscript was written through contributions of all authors.

Funding: This research received no external funding.

Acknowledgments: The authors would like to thank the Estonian Ministry of Education and Research (Grants No. IUT 33-20 and 19-32) for financial support.

Conflicts of Interest: The authors declare no conflict of interest.

\section{References}

1. Nanda, S.; Azargohar, R.; Dalai, A.K.; Kozinski, J.A. An assessment on the sustainability of lignocellulosic biomass for biorefining. Renew. Sustain. Energy Rev. 2015, 50, 925-941. [CrossRef]

2. Sanderson, K. Lignocellulose: A chewy problem. Nature 2011, 474, S12-S14. [CrossRef] [PubMed]

3. Choudhary, V.; Mushrif, S.H.; Ho, C.; Anderko, A.; Nikolakis, V.; Marinkovic, N.S.; Frenkel, A.I.; Sandler, S.I.; Vlachos, D.G. Insights into the Interplay of Lewis and Brønsted Acid Catalysts in Glucose and Fructose Conversion to 5-(Hydroxymethyl)furfural and Levulinic Acid in Aqueous Media. J. Am. Chem. Soc. 2013, 135, 3997-4006. [CrossRef] [PubMed]

4. Ruiz-Matute, A.I.; Hernández-Hernández, O.; Rodríguez-Sánchez, S.; Sanz, M.L.; Martínez-Castro, I. Derivatization of carbohydrates for GC and GC-MS analyses. J. Chromatogr. B Anal. Technol. Biomed. Life Sci. 2011, 879, 1226-1240. [CrossRef] [PubMed]

5. Chen, J.; Wang, S.; Huang, J.; Chen, L.; Ma, L.; Huang, X. Conversion of cellulose and cellobiose into sorbitol catalyzed by ruthenium supported on a polyoxometalate/metal-organic framework hybrid. ChemSusChem 2013, 6, 1545-1555. [CrossRef] [PubMed]

6. To, C.R. Cutting-edge research for a greener sustainable future. Green Chem. 2013, 15, 550-583. [CrossRef]

7. Eminov, S.; Filippousi, P.; Brandt, A.; Wilton-Ely, J.; Hallett, J. Direct Catalytic Conversion of Cellulose to 5-Hydroxymethylfurfural Using Ionic Liquids. Inorganics 2016, 4, 32. [CrossRef]

8. Zakrzewska, M.E.; Bogel-łukasik, E.; Bogel-łukasik, R. Ionic Liquid-Mediated Formation of 5-Hydroxymethylfurfural-A Promising Biomass-Derived Building Block. Chem. Rev. 2010, 111, $397-417$. [CrossRef] [PubMed]

9. Hyvärinen, S.; Mikkola, J.P.; Murzin, D.Y.; Vaher, M.; Kaljurand, M.; Koel, M. Sugars and sugar derivatives in ionic liquid media obtained from lignocellulosic biomass: Comparison of capillary electrophoresis and chromatographic analysis. Catal. Today 2014, 223, 18-24. [CrossRef]

10. Rinaldi, R.; Palkovits, R.; Schüth, F. Depolymerization of Cellulose Using Solid Catalysts in Ionic Liquids. Angew. Chem. 2008, 120, 8047-8050. [CrossRef] [PubMed]

11. Zhao, H.; Holladay, J.E.; Brown, H.; Zhang, Z.C. Metal Chlorides in Ionic Liquid Solvents Convert Sugars to 5-Hydroxymethylfurfural. Science 2007, 316, 1597-1601. [CrossRef] [PubMed]

12. Binder, J.B.; Raines, R.T. Simple Chemical Transformation of Lignocellulosic Biomass into Furans for Fuels and Chemicals. J. Am. Chem. Soc. 2009, 131, 1979-1985. [CrossRef] [PubMed] 
13. Zhang, Y.; Du, H.; Qian, X.; Chen, E.Y. Ionic Liquid-Water Mixtures: Enhanced $K_{\mathrm{w}}$ for Efficient Cellulosic Biomass Conversion. Energy Fuels 2010, 24, 2410-2417. [CrossRef]

14. Parveen, F.; Patra, T.; Upadhyayula, S. A structure-Activity relationship study using DFT analysis of Bronsted-Lewis acidic ionic liquids and synergistic effect of dual acidity in one-pot. New J. Chem. 2018, 42, 1423-1430. [CrossRef]

15. Matsagar, B.M.; Hossain, S.A.; Islam, T.; Alamri, H.R. Direct Production of Furfural in One-pot Fashion from Raw Biomass Using Brønsted Acidic Ionic Liquids. Sci. Rep. 2017, 7, 13508. [CrossRef] [PubMed]

16. Li, J.; Jing, Y.; Liu, C.; Zhang, D. A theoretical elucidation: Why does a $\mathrm{SO}_{3} \mathrm{H}$-functionalized imidazolium-based ionic liquid catalyze the conversion of 5-hydroxymethylfurfural to levulinic acid? New J. Chem. 2017, 41, 8714-8720. [CrossRef]

17. Du, X.; Zhang, J.; Wang, Y.; Qu, Y. Conversion of Carbohydrates into Platform Chemicals Catalyzed by Alkaline Ionic Liquids. Catalysts 2017, 7, 245. [CrossRef]

18. Omwoma, S.; Gore, C.T.; Ji, Y.; Hu, C.; Song, Y. Environmentally benign polyoxometalate materials. Coord. Chem. Rev. 2015, 286, 17-29. [CrossRef]

19. Weiping, W.Y.D.; Qinghong, Z. Polyoxometalates as efficient catalysts for transformations of cellulose into platform chemicals. Dalt. Trans. 2012, 41, 9817-9831. [CrossRef]

20. Chen, J.; Zhao, G.; Chen, L. Efficient production of 5-hydroxymethylfurfural and alkyl levulinate from biomass carbohydrate using ionic liquid-based polyoxometalate salts. RSC Adv. 2014, 4, 4194-4202. [CrossRef]

21. Abia, J.A.; Ozer, R. Development of polyoxometalate-ionic liquid compounds for processing cellulosic biomass. BioResources 2013, 8, 2924-2933. [CrossRef]

22. Chidambaram, M.; Bell, A.T. A two-step approach for the catalytic conversion of glucose to 2,5-dimethylfuran in ionic liquids. Green Chem. 2010, 12, 1253-1262. [CrossRef]

23. Aid, T.; Paist, L.; Lopp, M.; Kaljurand, M.; Vaher, M. An optimized capillary electrophoresis method for the simultaneous analysis of biomass degradation products in ionic liquid containing samples. J. Chromatogr. A 2016, 1447, 141-147. [CrossRef] [PubMed]

24. Aid, T.; Hyvärinen, S.; Vaher, M.; Koel, M.; Mikkola, J.P. Saccharification of lignocellulosic biomasses via ionic liquid pretreatment. Ind. Crop. Prod. 2016, 92, 336-341. [CrossRef]

25. Megías-sayago, C.; Álvarez, E. Epimerization of glucose over ionic liquid/Phosphomolybdate hybrids: Structure-Activity relationship. Green Chem. 2018, 20, 1042-1049. [CrossRef]

26. Bermejo-Deval, R.; Orazov, M.; Gounder, R.; Hwang, S.J.; Davis, M.E. Active sites in Sn-beta for glucose isomerization to fructose and epimerization to mannose. ACS Catal. 2014, 4, 2288-2297. [CrossRef]

27. Bermejo-Deval, R.; Assary, R.S.; Nikolla, E.; Moliner, M.; Roman-Leshkov, Y.; Hwang, S.J.; Palsdottir, A.; Silverman, D.; Lobo, R.F.; Curtiss, L.A.; et al. Metalloenzyme-like catalyzed isomerizations of sugars by Lewis acid zeolites. Proc. Natl. Acad. Sci. USA 2012, 109, 9727-9732. [CrossRef] [PubMed]

28. Gounder, R.; Davis, M.E. Monosaccharide and disaccharide isomerization over Lewis acid sites in hydrophobic and hydrophilic molecular sieves. J. Catal. 2013, 308, 176-188. [CrossRef]

29. Zhou, L.; Liang, R.; Ma, Z.; Wu, T.; Wu, Y. Conversion of cellulose to HMF in ionic liquid catalyzed by bifunctional ionic liquids. Bioresour. Technol. 2013, 129, 450-455. [CrossRef] [PubMed]

30. Bali, S.; Tofanelli, M.A.; Ernst, R.D.; Eyring, E.M. Chromium (III) catalysts in ionic liquids for the conversion of glucose to 5-(hydroxymethyl)furfural (HMF): Insight into metal catalyst:ionic liquid mediated conversion of cellulosic biomass to biofuels and chemicals. Biomass Bioenergy 2012, 42, 224-227. [CrossRef]

31. Tadesse, H.; Luque, R. Advances on biomass pretreatment using ionic liquids: An overview. Energy Environ. Sci. 2011, 4, 3913. [CrossRef]

32. Li, G.; Pidko, E.A.; Hensen, E.J.M. A Periodic DFT Study of Glucose to Fructose Isomerization on Tungstite $\left(\mathrm{WO}_{3} \mathrm{H}_{2} \mathrm{O}\right)$ : Influence of Group IV-VI Dopants and Cooperativity with Hydroxyl Groups. ACS Catal. 2016, 6, 4162-4169. [CrossRef]

33. Ju, F.; Vandervelde, D.; Nikolla, E. Molybdenum-Based Polyoxometalates as Highly Active and Selective Catalysts for the Epimerization of Aldoses. ACS Catal. 2014, 4, 1358-1364. [CrossRef]

34. Nguyen, H.; Nikolakis, V.; Vlachos, D.G. Mechanistic Insights into Lewis Acid Metal Salt-Catalyzed Glucose Chemistry in Aqueous Solution. ACS Catal. 2016, 6, 1497-1504. [CrossRef]

35. Zhou, C.; Zhao, J.; Elgasim, A.; Yagoub, A.; Ma, H. Conversion of glucose into 5-hydroxymethylfurfural in different solvents and catalysts: Reaction kinetics and mechanism. Egypt. J. Pet. 2017, 26, 477-487. [CrossRef] 
36. Weingarten, R.; Tae, Y.; Tompsett, G.A.; Fernández, A.; Sung, K.; Hagaman, E.W.; Curt, W.; Dumesic, J.A.C., Jr.; Huber, G.W. Conversion of glucose into levulinic acid with solid metal (IV) phosphate catalysts. J. Catal. 2013, 304, 123-134. [CrossRef]

37. Torres, A.I.; Daoutidis, P.; Tsapatsis, M. Continuous production of 5-hydroxymethylfurfural from fructose: A design case study. Energy Environ. Sci. 2010, 3, 1560. [CrossRef]

38. Ivanova, S. Hybrid organic-inorganic materials based on polyoxometalates. ISRN Chem. Eng. 2014, 2014, 963792. [CrossRef]

(c)

(C) 2018 by the authors. Licensee MDPI, Basel, Switzerland. This article is an open access article distributed under the terms and conditions of the Creative Commons Attribution (CC BY) license (http:/ / creativecommons.org/licenses/by/4.0/). 